\title{
Necessary condition for optimal control problem with interval-valued objective function
}

\author{
Ulcilea A. Severino Leal*, $\quad$ Geraldo N. Silva ${ }^{\dagger}$, \\ *Universidade Federal de Mato Grosso do Sul, CPCS, \\ 79560-000, Chapadão do Sul , MS \\ ${ }^{\dagger}$ Depto de Matemática Aplicada, Universidade Estadual Paulista (UNESP) \\ 15054-000, São José do Rio Preto, SP \\ E-mail: ulcilea.leal@ufms.br, gsilva@ibilce.unesp.br, \\ Weldon A. Lodwick \\ UCDenver - Department of Mathematics \\ Denver, Colorado, USA \\ E-mail: Weldon.Lodwick@ucdenver.edu
}

\begin{abstract}
This paper considers optimal control problems with interval-valued objective function. For this, we consider two concepts of order relation on the interval space to obtain necessary condition for this problem. We obtain the necessary conditions using the concept of generalized Hukuhara derivative ( $g H$-derivative) for interval-valued functions.
\end{abstract}

Keywords: Interval Analysis, Optimal Control, Generalized Hukuhara Derivative

\section{Introduction}

The optimal control problems in general are assumed to have deterministic coefficients and fixed values. However, there are many situations where this assumption is not valid, and the problem involves some uncertainty. Thus, decision-making methods under uncertainty are required. The stochastic and the fuzzy programming were proposed to deal with this situation (see $[3,12])$. Whereas the probability and the membership functions play important roles in each method, it is not always easy for the decision maker to specify them.

This paper proposes a simple decision making method under uncertainty for optimal control problem. The optimal control problem to study is

$$
\begin{aligned}
& \min I(x, u)=\int_{t_{0}}^{t_{1}} L(t, x(t), u(t)) d t \\
& \text { subject to: } u(t) \in U_{a d} \\
& x^{\prime}(t)=f(t, x(t), u(t)), \quad t \in\left(t_{0}, t_{1}\right) \\
& x\left(t_{0}\right)=\mathbf{x}_{0},
\end{aligned}
$$

where $L(t, x(t), u(t))=[\underline{l}(t, x(t), u(t)), \bar{l}(t, x(t), u(t))] ; \underline{l}(t, x(t), u(t)) \leq \bar{l}(t, x(t), u(t))$ is the interval-valued objective function, $x(t)$ is the state equation, $u(t)$ is the control function and $\mathbf{x}_{0}$ is the initial condition of the problem.

Sufficient conditions for optimization problems via gH-derivative were proposed by ChalcoCano et al. [4]. Wu [10] proposed sufficient conditions via the Hukuhara derivative and later $\mathrm{Wu}$

${ }^{*} \dagger$ The research in this paper has been partially supported by both FAPESP-Brazil under the grants (2012/00189-3 and CEPID 2013/07375-0 of Industrial Mathematics) and CNPq under the grants (309335/20124 and $479109 / 2013-3)$. 
[11] derived the KKT conditions for the resulting multiobjective programming problem. The conditions proposed by Chalco-Cano et al. [4] are more general because they used a concept of differentiability which is more general than H-differentiability.

This paper obtains necessary condition to optimal control problem with interval-valued objective function considering order relationships between two closed intervals. The necessary condition is obtained using gH-derivative of interval-valued function.

\section{Interval arithmetic}

This section describes the basic elements of the interval arithmetic and the generalized Hukuhara difference proposed in Stefanini [7] and used by Stefanini and Bede [8] to define the generalized Hukurara derivative for the case of interval-valued functions. Let $\mathcal{K}_{C}^{n}$ be a space of nonempty compact and convex set of $\mathbb{R}^{n}$. If $n=1 \mathbb{I}(\mathbb{R})$ denotes the set of bounded closed intervals of the real line, i.e., $\mathbb{I}(\mathbb{R})=\{[\underline{a}, \bar{a}] ; \underline{a}, \bar{a} \in \mathbb{R}$ and $\underline{a} \leq \bar{a}\}$, where $\underline{a}$ and $\bar{a}$ means the lower and upper bounds of any interval $A \in \mathbb{I}(\mathbb{R})$, respectively. Given two intervals $A=[\underline{a}, \bar{a}], B=[\underline{b}, \bar{b}] \in \mathbb{I}(\mathbb{R})$ and $k \in \mathbb{R}$ we consider the following operations

$$
\begin{aligned}
& A+B=[\underline{a}+\bar{b}, \bar{a}+\bar{b}], \\
& k \cdot A=\left\{\begin{array}{l}
{[k \cdot \underline{a}, k \cdot \bar{a}] \text { if } k \geq 0} \\
{[k \cdot \bar{a}, k \cdot \underline{a}] \text { if } k \leq 0}
\end{array},\right.
\end{aligned}
$$

then $A-B=A+(-1) B=[\underline{a}-\bar{b}, \bar{a}-\underline{b}]$. However, $\mathbb{I}(\mathbb{R})$ with these operations is not a linear space since there are no additive inverses, and therefore subtraction is not well defined (see [1]). Hukuhara [5] defined the Hukuhara difference (H-difference) for two intervals. This difference is unique, but it does not always exist. A generalization of the Hukuhara difference was introduced by Stefanini and Bede [8] to always have a difference.

Definition 1. [8] The generalized Hukuhara difference ( $g$ H-difference, for short) of two intervals $A, B \in \mathbb{I}(\mathbb{R})$ is defined as follows

$$
A \ominus_{g} B=C\left\{\begin{array}{l}
(a) \text { if } A=B+C \text { or } \\
(b) \text { if } B=A+(-1) C
\end{array} .\right.
$$

In case $(a)$, the gH-difference and H-difference are equal. For gH-difference, since $A \ominus_{g} A=[0,0]$, the gH-difference of the intervals $A=[\underline{a}, \bar{a}]$ and $B=[\underline{b}, \bar{b}]$ always exist, and it is equal to $A \ominus_{g} B=[\min \{\underline{a}-\underline{b}, \bar{a}-\bar{b}\}, \max \{\underline{a}-\underline{b}, \bar{a}-\bar{b}\}]$. An interval-valued function $F:\left[t_{0}, t_{1}\right] \rightarrow \mathbb{I}(\mathbb{R})$ is denoted by $F(t)=[\underline{f}(t), \bar{f}(t)]$ such that $\underline{f}(t) \leq \bar{f}(t)$ for all $t \in\left[t_{0}, t_{1}\right]$. The functions $\underline{f}(t)$ and $\bar{f}(t)$ will be called, respectively, the lower and upper functions of $F$. Based in the gH-difference concept Stefanini and Bede [8] proposed the following definition.

Definition 2. [8] Let $t \in] t_{0}, t_{1}[$ and $h$ be such that $t+h \in] t_{0}, t_{1}[$, then the generalized Hukuhara derivative of an interval-valued function $F:] t_{0}, t_{1}[\rightarrow \mathbb{I}(\mathbb{R})$ at $t$ is defined as

$$
F^{\prime}(t)=\lim _{h \rightarrow 0} \frac{F(t+h) \ominus_{g} F(t)}{h} .
$$

If $F^{\prime}(t) \in \mathbb{I}(\mathbb{R})$ satisfying (2) exists, then $F$ is said to be generalized Hukuhara differentiable (gH-differentiable for short) at $t . F$ is gH-differentiable on $] t_{0}, t_{1}[$ if $F$ is gH-differentiable at each point $t \in] t_{0}, t_{1}[$.

Theorem 2.1. [8] Let $F:] t_{0}, t_{1}[\rightarrow \mathbb{I}(\mathbb{R})$ be an interval-valued function such that $F(t)=[\underline{f}(t), \bar{f}(t)]$. Then $F$ is $g H$-differentiable at $t$ if and only if one of the following cases holds

1. $\underline{f}$ and $\bar{f}$ are differentiable at $t$. 
2. [4] Lateral derivatives exist and satisfy $(f)_{-}^{\prime}(t)=(\bar{f})_{+}^{\prime}(t)$ and $(f)_{+}^{\prime}(t)=(\bar{f})_{-}^{\prime}(t)$.

Furthermore,

$$
F^{\prime}(t)=\left[\min \left\{\underline{f}^{\prime}(t), \bar{f}^{\prime}(t)\right\}, \max \left\{\underline{f}^{\prime}(t), \bar{f}^{\prime}(t)\right\}\right] .
$$

Theorem 2.2. [4] Let $F:] t_{0}, t_{1}[\rightarrow \mathbb{I}(\mathbb{R})$ be an interval-valued function such that $F(t)=[\underline{f}(t), \bar{f}(t)]$. If $F$ is a gH-differentiable interval-valued function at $t \in] t_{0}, t_{1}[$. Then, $f+\bar{f}$ is a differentiable function at $t$ (in the classical sense).

Theorem 2.3. [9] If $F:] t_{0}, t_{1}[\rightarrow \mathbb{I}(\mathbb{R})$ is gH-differentiable interval-valued function at $t \in] t_{0}, t_{1}[$ then it is continuous at $t$.

Consider the interval-valued function $F$ defined on $\mathbb{R}^{n}$, i.e., $F(\mathbf{x})=F\left(x_{1}, x_{2}, \ldots, x_{n}\right) \in \mathbb{I}(\mathbb{R})$ for each $\mathbf{x}=\left(x_{1}, x_{2}, \ldots x_{n}\right) \in \mathbb{R}^{n}$, where $\underline{f}(\mathbf{x})=\underline{f}\left(x_{1}, x_{2}, \ldots, x_{n}\right)$ and $\bar{f}(\mathbf{x})=\bar{f}\left(x_{1}, x_{2}, \ldots, x_{n}\right)$ are defined on $\mathbb{R}^{n}$. Since $\left(\mathcal{K}_{C}, H\right)$ is a metric space, then we can define continuity of an interval valued function $F: \mathbb{R}^{n} \rightarrow \mathbb{I}(\mathbb{R})$ at $\mathbf{x}_{0} \in \mathbb{R}^{n}$.

Proposition 2.1. [1] Let $F$ be an interval-valued function defined on $X \subset \mathbb{R}^{n}$ and $\boldsymbol{x}_{0} \in X$. Then $F$ is continuous at $\boldsymbol{x}_{0}$ if and only if $\underline{f}$ and $\bar{f}$ are continuous at $\boldsymbol{x}_{0}$.

Chalco-Cano et al. [4] defined the partial derivative of the interval-valued function $F$ via gH-derivative.

Definition 3. [4] Let $F$ be an interval-valued function defined on $X \subset \mathbb{R}^{n}$ and let $\boldsymbol{x}_{0}=$ $\left(x_{1}^{0}, x_{2}^{0}, \ldots, x_{n}^{0}\right)$ be an element of $X$ fixed. Consider the interval-valued function

$$
h_{j}\left(x_{j}\right)=F\left(x_{1}^{0}, \ldots, x_{j-1}^{0}, x_{j}, x_{j+1}^{0}, \ldots, x_{n}^{0}\right),
$$

for $j=1, \ldots, n$. If $h_{j}$ is $g H$-differentiable at $x_{j}^{0}$, then $F$ has the $j$ th partial $g H$-derivative at $\boldsymbol{x}_{0}$, $\left(\left(\frac{\partial F}{\partial x_{j}}\right)_{g}\left(x_{0}\right)\right)$, and $\left(\left(\frac{\partial F}{\partial x_{j}}\right)_{g}\left(x_{0}\right)\right)=\left(h_{j}\right)^{\prime}\left(\boldsymbol{x}_{j}^{0}\right)$.

Definition 4. [4] Let $F$ be an interval-valued function defined on $X \subset \mathbb{R}^{n}$ and $\boldsymbol{x}_{0}=\left(x_{1}^{0}, x_{2}^{0}, \ldots, x_{n}^{0}\right)$ be a fixed element of $X . F$ is continuously gH-differentiable at $\boldsymbol{x}_{0}$ if all the partial gH-derivatives exist on some neighborhoods of $\boldsymbol{x}_{0}$ and are continuous at $\boldsymbol{x}_{0}$.

Proposition 2.2. [4] Let $F$ be an interval-valued function defined on $X \subset \mathbb{R}^{n}$. If $F$ is continuously gH-differentiable at $\boldsymbol{x}_{0}$, then $\underline{f}+\bar{f}$ is continuously differentiable at $\boldsymbol{x}_{0}$ (in the classical sense).

\section{Interval-valued optimal control problem}

This section briefly presents the interval optimal control problems and analyzes it. The optimal control problem described above is the following:

$$
\begin{aligned}
& \min I(x, u)=\int_{t_{0}}^{t_{1}} L(t, x(t), u(t)) d t \\
& \text { subject to: } u(t) \in U_{a d} \\
& x^{\prime}(t)=f(t, x(t), u(t)), \quad t \in\left(t_{0}, t_{1}\right) \\
& x\left(t_{0}\right)=\mathbf{x}_{0},
\end{aligned}
$$

where $f:\left[t_{0}, t_{1}\right] \times \mathbb{R}^{n} \times \mathbb{R}^{m} \rightarrow \mathbb{R}^{n}$ is a classical function, $L: \mathbb{R} \times \mathbb{R}^{n} \times \mathbb{R}^{m} \rightarrow I(\mathbb{R})$ is the intervalvalued objective function, $x(t)$ is the state variable, $u(t)$ is the control variable, where $x(t)$ and $u(t)$ are related via the problem dynamics, $x^{\prime}(t)=f(t, x(t), u(t)), \quad t \in\left(t_{0}, t_{1}\right), x\left(t_{0}\right)=\mathbf{x}_{0}$, and 
$u(t) \in U_{a d}$, the set of admissible controls functions. If restrictions on the control variables are not made, the set of admissible controls is given by $U_{a d}=C\left(\left[t_{0}, t_{1}\right] ; \mathbb{R}^{m}\right)$. For the problem described above the admissible trajectory set is given by

$$
X_{a d}:=\left\{x(t) \in C\left(\left[t_{0}, t_{1}\right] ; \mathbb{R}^{n}\right) ; x^{\prime}(t)=f(t, x(t), u(t)), u \in U_{a d}, \quad x\left(t_{0}\right)=\mathbf{x}_{0}\right\} .
$$

The candidate solutions of the problem (3) are the admissible processes $(x(t), u(t)) \in X_{a d} \times U_{a d}$.

We denote by $L(t, x(t), u(t))=[\underline{l}(t, x(t), u(t)), \bar{l}(t, x(t), u(t))]$, with $\underline{l}(t, x(t), u(t)) \leq \bar{l}(t, x(t), u(t))$ for all $t \in\left[t_{0}, t_{1}\right]$. Using Aumann [2], we have that

$$
\int_{t_{0}}^{t_{1}} L(t, x(t), u(t)) d t=\left[\int_{t_{0}}^{t_{1}} \underline{l}(t, x(t), u(t)) d t, \int_{t_{0}}^{t_{1}} \bar{l}(t, x(t), u(t)) d t\right] .
$$

We will interpret a solution of problem (3) with a partial order relation in the space $\mathbb{I}(\mathbb{R})$. This is similar to the Pareto optimal solution concept used in the multiobjective programming problem (see $[6,10,4]$. We study the problem using one order relation and compare to the other two order relations. First, we present the usual order relation, $\leq_{L U}$.

Let $A=[\underline{a}, \bar{a}]$ and $B=[\underline{b}, \bar{b}]$ be two intervals. The order relation $\leq_{L U}$ is defined by

$$
A \leq_{L U} B \text { if and only if } \underline{a} \leq \underline{b} \text { and } \bar{a} \leq \bar{b},
$$

and $A<_{L U} B$ if and only if $A \leq_{L U} B$ and $A \neq B$.

Let $\left(x^{*}(t), u^{*}(t)\right)$ be an admissible process of problem (3), i.e., $\left(x^{*}(t), u^{*}(t)\right) \in X_{a d} \times U_{a d}$. We say that $\left(x^{*}(t), u^{*}(t)\right)$ is an optimal LU-solution of problem (3) if there exist no $(x(t), u(t)) \in$ $X_{a d} \times U_{a d}$ such that $I(x(t), u(t))<_{L U} I\left(x^{*}(t), u^{*}(t)\right)$.

By width $a^{S}$, we mean

$$
a^{S}=(\bar{a}-\underline{a}) .
$$

The order relation $\leq_{L S}$ is defined by 1 ) for maximization

$$
A \geq_{L S} B \text { if and only if } \bar{a} \geq \bar{b} \text { and } a^{S} \leq b^{S},
$$

and $A>_{L S} B$ if and only if $A \geq_{L S} B$ and $A \neq B$. 2) for minimization

$$
A \leq_{L S} B \text { if and only if } \underline{a} \leq \underline{b} \text { and } a^{S} \leq b^{S},
$$

and $A<_{L S} B$ if and only if $A \leq_{L S} B$ and $A \neq B$.

Let $\left(x^{*}(t), u^{*}(t)\right)$ be an admissible process of problem (3), i.e., $\left(x^{*}(t), u^{*}(t)\right) \in X_{a d} \times U_{a d}$. We say that $\left(x^{*}(t), u^{*}(t)\right)$ is an optimal LS-solution of problem (3) if there exists no $(x(t), u(t)) \in$ $X_{a d} \times U_{a d}$ such that $I(x(t), u(t))<_{L S} I\left(x^{*}(t), u^{*}(t)\right)$.

Proposition 3.1. [4] Let $A=[\underline{a}, \bar{a}]$ and $B=[\underline{b}, \bar{b}]$ be two intervals in $\mathbb{I}(\mathbb{R})$. If $A \leq_{L S}$ $B$ then $A \leq_{L U} B$.

Theorem 3.1. Let $\left(x^{*}(t), u^{*}(t)\right)$ be an admissible process of problem (3), i.e., $\left(x^{*}(t), u^{*}(t)\right) \in$ $X_{a d} \times U_{a d}$. If $\left(x^{*}(t), u^{*}(t)\right)$ is an optimal LU-solution of problem (3) then it is an optimal LS-solution of problem (3).

Proof. Suppose that $\left(x^{*}(t), u^{*}(t)\right)$ is not an optimal LS-solution of problem (3). Then there exists $(x(t), u(t)) \in X_{a d} \times U_{a d}$ such that $I(x(t), u(t)) \leq_{L S} I\left(x^{*}(t), u^{*}(t)\right)$ and $I(x(t), u(t)) \neq$ $I\left(x^{*}(t), u^{*}(t)\right)$. From Proposition (3.1) $I(x(t), u(t)) \leq_{L U} I\left(x^{*}(t), u^{*}(t)\right)$ and $I(x(t), u(t)) \neq$ $I\left(x^{*}(t), u^{*}(t)\right)$, which is a contradiction with the hypothesis of the Theorem.

A similar result is valid for the case of maximizing. In this case, we have that: let $\left(x^{*}(t), u^{*}(t)\right)$ be an admissible process of maximization problem, i.e., $\left(x^{*}(t), u^{*}(t)\right) \in X_{a d} \times U_{a d}$. If $\left(x^{*}(t), u^{*}(t)\right)$ is an optimal LU-solution of the maximization problem then it is an optimal LS-solution for the maximization case. 
Definition 5. [4] Let $F$ be an interval-valued function defined on a convex set $X \subset \mathbb{R}^{n}$. Then

1. F is $L U$-convex at $\boldsymbol{x}^{*}$ if

$$
F\left(\lambda \boldsymbol{x}^{*}+(1-\lambda) \boldsymbol{x}\right) \leq_{L U} \lambda F\left(\boldsymbol{x}^{*}\right)+(1-\lambda) F(\boldsymbol{x})
$$

for all $\lambda \in(0,1)$ and each $\boldsymbol{x} \in X$.

2. $F$ is LS-convex at $\boldsymbol{x}^{*}$ if

$$
F\left(\lambda \boldsymbol{x}^{*}+(1-\lambda) \boldsymbol{x}\right) \leq_{L S} \lambda F\left(\boldsymbol{x}^{*}\right)+(1-\lambda) F(\boldsymbol{x})
$$

for all $\lambda \in(0,1)$ and each $\boldsymbol{x} \in X$.

Proposition 3.2. [4] Let $X$ be a convex subset of $\mathbb{R}^{n}$, and $F$ be an interval-valued function defined on $X$. Then it has following properties

1. $F$ is $L U$-convex at $\boldsymbol{x}^{*}$ if and only if $\underline{f}$ and $\bar{f}$ are convex at $\boldsymbol{x}^{*}$.

2. F is LS-convex at $\boldsymbol{x}^{*}$ if and only if $\underline{f}$ and $f^{S}$ are convex at $\boldsymbol{x}^{*}$.

3. If $F$ is $L S$-convex at $\boldsymbol{x}^{*}$ then $F$ is $L U$-convex at $\boldsymbol{x}^{*}$.

\section{Necessary condition for optimal control problem}

This section obtains necessary condition for optimal control problem using gH-differentiable of interval-valued objective function. Next, we define an interval-valued auxiliar function that is called interval-valued Hamiltonian function.

Definition 6. Let $H:\left[t_{0}, t_{1}\right] \times \mathbb{R}^{n} \times \mathbb{R}^{m} \times \mathbb{R}^{n} \rightarrow I(\mathbb{R})$ be an interval-valued function. Then $H$ defined by

$$
H(t, x(t), u(t), \lambda(t))=L(t, x(t), u(t))+p^{T}(t) f(t, x(t), u(t)),
$$

where $p(t) \in C\left(\left[t_{0}, t_{1}\right] ; \mathbb{R}^{n}\right)$ is continuously differentiable differentiable, is called the intervalvalued Hamiltonian function.

Next, we present necessary condition in the interval-valued form for the gH-differentiable case. The following result is a necessary condition for control problem (3) based on the concept of gH-differentiable functions. This result is a variant of the maximum principle for the case that $L$ and $f$ are LU-convex and convex, respectively.

Theorem 4.1. Suppose that the interval-valued objetive function $L:\left[t_{0}, t_{1}\right] \times \mathbb{R}^{n} \times \mathbb{R}^{m} \rightarrow$ $I(\mathbb{R})$ is continuously $g H$-differentiable and $L U$-convex in the variable $u(t)$ over $\left[t_{0}, t_{1}\right]$. Let $f:\left[t_{0}, t_{1}\right] \times \mathbb{R}^{n} \times \mathbb{R}^{m} \rightarrow \mathbb{R}^{n}$ be continuously differentiable and convex in the variable $u(t)$ on $\left[t_{0}, t_{1}\right]$. If $\left(x^{*}(t), u^{*}(t)\right)$ is an optimal LU-solution of problem (3) on $X_{a d} \times U_{\text {ad }}$ subject to $x^{\prime}(t)=f(t, x(t), u(t))$, then there exists a multiplier $p^{*} \in C^{1}\left(\left[t_{0}, t_{1}\right] ; \mathbb{R}^{n}\right)$ such that

$$
\left\{\begin{array}{l}
x^{\prime}(t)=\frac{\partial}{\partial p}\left(\frac{\underline{h}+\bar{h}}{2}\right)\left(t, x, u, p^{*}\right) \\
p^{\prime}(t)=-\frac{\partial}{\partial x}\left(\frac{\underline{h}+\bar{h}}{2}\right)\left(t, x, u, p^{*}\right) \\
x\left(t_{0}\right)=x_{0}
\end{array} .\right.
$$

Furthermore, the control $u^{*}(t)$ satisfies the following condition maximum

$$
(\underline{h}+\bar{h})\left(t, x^{*}(t), u^{*}(t), p^{*}(t)\right)=\min _{u(t)}\left\{(\underline{h}+\bar{h})\left(t, x^{*}(t), u(t), p^{*}(t)\right)\right\} \quad t \in\left(t_{0}, t_{1}\right) .
$$


Proof. If $\left(x^{*}(t), u^{*}(t)\right)$ is an optimal LU-solution process, then there exists no $(x(t), u(t)) \in$ $X_{a d} \times U_{a d}$ subject to $x^{\prime}(t)=f(t, x(t), u(t))$ such that $I(x, u)<_{L U} I\left(x^{*}, u^{*}\right)$. Thus,

$$
\begin{gathered}
\left\{\begin{array} { l } 
{ \int _ { t _ { 0 } } ^ { t _ { 1 } } \underline { l } ( x , u ) d t < \int _ { t _ { 0 } } ^ { t _ { 1 } } \underline { l } ( x ^ { * } , u ^ { * } ) d t } \\
{ \int _ { t _ { 0 } } ^ { t _ { 1 } } \overline { l } ( x , u ) d t \leq \int _ { t _ { 0 } } ^ { t _ { 1 } } \overline { l } ( x ^ { * } , u ^ { * } ) d t }
\end{array} \quad \text { or } \left\{\begin{array}{l}
\int_{t_{0}}^{t_{1}} \underline{l}(x, u) d t \leq \int_{t_{0}}^{t_{1}} \underline{l}\left(x^{*}, u^{*}\right) d t \\
\int_{t_{0}}^{t_{1}} \bar{l}(x, u) d t<\int_{t_{0}}^{t_{1}} \bar{l}\left(x^{*}, u^{*}\right) d t
\end{array},\right.\right. \\
\text { or }\left\{\begin{array}{l}
\int_{t_{0}}^{t_{1}} \underline{l}(x, u) d t<\int_{t_{0}}^{t_{1}} \underline{l}\left(x^{*}, u^{*}\right) d t \\
\int_{t_{0}}^{t_{1}} \bar{l}(x, u) d t<\int_{t_{0}}^{t_{1}} \bar{l}\left(x^{*}, u^{*}\right) d t
\end{array} .\right.
\end{gathered}
$$

Therefore,

$$
\int_{t_{0}}^{t_{1}}\left(\frac{\underline{l}+\bar{l}}{2}\right)(x, u) d t<\int_{t_{0}}^{t_{1}}\left(\frac{\underline{l}+\bar{l}}{2}\right)\left(x^{*}, u^{*}\right) d t
$$

Then, $\left(x^{*}, u^{*}\right)$ is an optimal process for the following problem:

$$
\begin{aligned}
& \min \int_{t_{0}}^{t_{1}}\left(\frac{\underline{l}+\bar{l}}{2}\right)(t, x(t), u(t)) d t \\
& \text { subject to: } \quad(x, u) \in X_{a d} \times U_{a d} \\
& x^{\prime}(t)=f(t, x(t), u(t)),
\end{aligned}
$$

where $\left(\frac{\underline{l}+\bar{l}}{2}\right)$ is continuously differentiable, because $L$ is continuously gH-differentiable and $f$ is continuously differentiable. Moreover, if $L$ is LU-convex then $\underline{l}$ and $\bar{l}$ are convex, and $f$ is convex, so the Hamiltonian function of this problem is given by

$$
\left(\frac{\underline{h}+\bar{h}}{2}\right)=\left(\frac{\underline{l}+\bar{l}}{2}\right)+p^{T} f
$$

This Hamiltonian (9) is convex at $u(t)$. By the classical maximum principle, there exists $p^{*} \in$ $C^{1}\left(\left[t_{0}, t_{1}\right] ; \mathbb{R}^{n}\right)$ such that the system (8) and the condition maximum are valid.

\section{Conclusion}

We considered two order relations on the space of intervals. We used the gH-derivative for interval-valued function to obtain necessary condition for optimal control problems with intervalvalued objective function obtaining the optimal solution. We also demonstrated the relations between LU-solution and the other definition of solution based on their respective order relations.

\section{References}

[1] J.P. Aubin and A. Cellina. Differential Inclusions. Springer-Verleg, New York, 1984.

[2] Robert J. Aumann. Integrals of set-valued functions. Journal of Mathematical Analysis and Applications, 12:1-12, 1965.

[3] R. E. Bellman and L. A. Zadeh. Decision-making in a fuzzy environment. Management Science, 17:141-164, 1970.

[4] Y. Chalco-Cano, A. Rufián-Lizana, H. Román-Flores, and M. D. Jiménez-Gamero. Calculus for interval-valued functions using generalized Hukuhara derivative and applications. Fuzzy Sets and Systems, 219:49-67, 2013. 
[5] M. Hukuhara. Intégration des applications mesurables dont la valeur est un compact convex. Funkcial Ekvac, 10:205-229, 1967.

[6] Hisao Ishibuchi and Hideo Tanaka. Multiobjective programming in optimization of the interval objetive function. European Journal of Operation Research, 48:219-225, 1990.

[7] L. Stefanini. A generalizetion of Hukuhara difference for interval and fuzzy arithmetic. Series on Advances in Soft Computating, 48, 2008.

[8] L. Stefanini and B. Bede. Generalized Hukuhara differentiability of interval-valued functions and interval differential equations. Nonlinear Analysis, 71:1311-1328, 2009.

[9] Luciano Stefanini and Barnabás Bede. Some notes on generalized Hukuhara differentiability of interval-valued functions and interval differential equations. Elsevier Science, 2012.

[10] H. C. Wu. The Karush-Kuhn-Tucker optimization conditions in an optimization problem with interval-valued objective function. European Journal of Operation Research, 176:4759, 2007.

[11] H. C. Wu. The Karush-Kuhn-Tucker optimality conditions in multiobjective programming problems with interval-valued objective functions. European Journal of Operation Research, 196:49-60, 2009.

[12] H. J. Zimmerman. Description and optimization of fuzzy systems. International Journal of General Systems, 2:206-215, 1976. 